\title{
QUENCHING OF NUMERICAL SOLUTIONS FOR SOME SEMILINEAR HEAT EQUATIONS WITH A VARIABLE REACTION
}

\author{
F. K. N'GOHISSE ${ }^{1}$
}

\begin{abstract}
In this paper, under certain conditions, we show that the solution of the semidiscrete form of a semilinear heat equation with a variable reaction is quenched in a finite time and estimate its semidiscrete quenching time. We also show that the semidiscrete quenching time converges to the continuous one when the mesh size goes to zero. In the same way, an analogous study has been investigated taking into account the discrete form of the above problem. Finally, we present some computational results.
\end{abstract}

2000 Mathematics Subject Classification: 35B40, 35B50, 35K60, 65M06.

Keywords: semidiscretizations, discretizations, semilinear heat equations, semidiscrete quenching time, discrete quenching time, convergence.

\section{Introduction}

We consider the following initial-boundary value problem for a semilinear parabolic equation of the form

$$
\begin{gathered}
u_{t}=u_{x x}-u^{-p(x)}, \quad x \in(0,1), \quad t \in(0, T), \\
u_{x}(0, t)=0, \quad u_{x}(1, t)=0, \quad t \in(0, T), \\
u(x, 0)=u_{0}(x)>0, \quad x \in[0,1],
\end{gathered}
$$

where $p \in C^{0}([0,1]), 0<u_{0}(x) \leqslant 1$ in $[0,1], 0<p_{*}=\inf _{x \in(0,1)} p(x) \leqslant \sup _{x \in(0,1)} p(x)=p^{*}$. The initial datum is $u_{0} \in C^{1}([0,1]), u_{0}(x)>0$ for $x \in[0,1], u_{0}^{\prime}(0)=0, u_{0}^{\prime}(1)=0$. Here $(0, T)$ is the maximal time interval of existence of the solution $u$. The time $T$ may be finite or infinite. When $T$ is infinite, we say that the solution $u$ exists globally. When $T$ is finite, then the solution $u$ develops a singularity in a finite time, namely

$$
\lim _{t \rightarrow T} u_{\min }(t)=0
$$

where $u_{\min }(t)=\min _{0 \leqslant x \leqslant 1} u(x, t)$. In this case, we say that the solution $u$ quenches in a finite time and the time $T$ is called the quenching time of the solution $u$. The theoretical study of the quenching of solutions for semilinear heat equations has been the subject of investigations of many authors (see $[2,3,10,14,21]$, and the references therein). In the case where $p(x)=p>0$, $p$ being a positive constant, the phenomenon of quenching has received extensive studies. However, to the best of our knowledge, the first paper that considered a problem with a

\footnotetext{
${ }^{1}$ Université d'Abobo-Adjamé, UFR-SFA, Département de Mathématiques et Informatiques, 01 BP 1003 Abidjan 01, Cóte d'Ivoire. E-mail: ngohisse@yahoo.fr
} 
variable reaction is that of Fereira et al. [8] where the authors studied the critical exponent. It is easy to check that if we set $f(x, u)=-u^{-p(x)}$, then we observe that the function $f$ is continuous in both variables and locally Lipschitz in the second one. Consequently, we can apply the maximum principle (see, for instance, [8]). In addition, by standard methods, one may easily prove the time-local existence and uniqueness of the classical solution (see [4,9]).

In this paper, we are interested in the numerical study using the semidiscrete and discrete forms of (1.1)-(1.3). We start by constructing a semidiscrete scheme as follows. Let $I$ be a positive integer and let us define the grid $x_{i}=i h, 0 \leqslant i \leqslant I$, where $h=1 / I$. Approximate the solution $u$ of $(1.1)-(1.3)$ by the solution $U_{h}(t)=\left(U_{0}(t), U_{1}(t), \ldots, U_{I}(t)\right)^{\top}$ of the following semidiscrete equations:

$$
\begin{gathered}
\frac{d U_{i}(t)}{d t}=\delta^{2} U_{i}(t)-U_{i}^{-p_{i}}(t), \quad 0 \leqslant i \leqslant I, \quad t \in\left(0, T_{b}^{h}\right), \\
U_{i}(0)=\varphi_{i}>0, \quad 0 \leqslant i \leqslant I,
\end{gathered}
$$

where $p_{i}$ is the approximation of $p\left(x_{i}\right), 0 \leqslant i \leqslant I$,

$$
\begin{gathered}
\delta^{2} U_{0}(t)=\frac{2 U_{1}(t)-2 U_{0}(t)}{h^{2}}, \quad \delta^{2} U_{I}(t)=\frac{2 U_{I-1}(t)-2 U_{I}(t)}{h^{2}}, \\
\delta^{2} U_{i}(t)=\frac{U_{i+1}(t)-2 U_{i}(t)+U_{i-1}(t)}{h^{2}}, \quad 1 \leqslant i \leqslant I-1 .
\end{gathered}
$$

Here, $\left(0, T_{b}^{h}\right)$ is the maximal time interval on which $U_{h \min }(t)>0$ where

$$
U_{h \min }(t)=\min _{0 \leqslant i \leqslant I} U_{i}(t)
$$

In the case where $T_{b}^{h}$ is finite, we say that the solution $U_{h}(t)$ is quenched in a finite time and the time $T_{b}^{h}$ is called the quenching time of the solution $U_{h}(t)$. In the present paper, we give some conditions under which the solution of (1.4)-(1.5) is quenched in a finite time and estimate its semidiscrete quenching time. We also show that the semidiscrete quenching time converges to the continuous one when the mesh size goes to zero. Similar results have also been obtained taking a discrete form of (1.1)-(1.3). Our work was motived by the papers in $[1,11,12,22]$ and [23]. In [11] and [23], the authors used semidiscrete forms for some semilinear heat equations to study the blow-up phenomenon. Recently, in [22], Nabongo and Boni handled the phenomenon of quenching using semidiscrete and discrete schemes. In this paper, we consider the same problem as in [22] and show that all properties obtained in the case of a constant exponent can be reproduced in the case of a variable reaction. The rest of the paper is organized as follows. In the next section, under certain conditions, we prove that the solution of the semidiscrete problem is quenched in a finite time and estimate its semidiscrete quenching time. In Section 3, we study the convergence of the semidiscrete quenching time. In Section 4, we approximate problem (1.1)-(1.3) by a discrete scheme and give some properties concerning the discrete scheme. In Section 5, we study the quenching for the discrete solution and estimate the numerical quenching time. In Section 6, we prove that the numerical quenching time converges to the real one when the mesh size tends to zero. Finally, in the last section we report on some numerical experiments using several discretisations of (1.1)-(1.3). 


\section{Quenching of semidiscrete solutions}

In this section under some assumptions, we show that the solution of the semidiscrete problem is quenched in a finite time and estimate its semidiscrete quenching time.

To simplify the text, we shall use the following notational conventions:

$$
U(t)=U_{i}(t), \quad \text { and } \quad \delta^{2} U(t)=\delta^{2} U_{i}(t)
$$

We first recall some results on the semidiscrete maximum principle.

The following lemma is a semidiscrete form of the maximum principle.

Lemma 2.1. Let $a_{h} \in C^{0}\left([0, T), \mathbb{R}^{I+1}\right)$ and let $V_{h} \in C^{1}\left([0, T), \mathbb{R}^{I+1}\right)$ be such that for $t \in(0, T)$

$$
\frac{d V(t)}{d t}-\delta^{2} V(t)+a(t) V(t) \geqslant 0, \quad V(0) \geqslant 0 .
$$

Then we have $V(t) \geqslant 0, t \in(0, T)$.

Proof. For the proof see [22].

Another version of the semidiscrete maximum principle is the following comparison lemma.

Lemma 2.2. Let $V_{h}, U_{h} \in C^{1}\left([0, T), \mathbb{R}^{I+1}\right)$ and $f \in C^{0}(\mathbb{R} \times \mathbb{R}, \mathbb{R})$ be such that for $t \in(0, T)$,

$$
\frac{d V(t)}{d t}-\delta^{2} V(t)+f(V(t), t)<\frac{d U(t)}{d t}-\delta^{2} U(t)+f(U(t), t) \quad V(0)<U(0) .
$$

Then we have $V_{h}(t)<U_{h}(t), t \in(0, T)$.

Proof. For the proof see [22].

To finish the recalls, let us state the result of the operator $\delta^{2}$.

Lemma 2.3. Let $U_{h} \in \mathbb{R}^{I+1}$ be such that $U_{h}>0$ and let $\gamma>0$. Then we have

$$
\delta^{2} U^{-\gamma} \geqslant-\gamma U^{-\gamma-1} \delta^{2} U
$$

Proof. See, for instance, [22].

We can handle now the main theorem of this section.

Theorem 2.1. Suppose that there exists a positive constant $A \in(0,1]$ such that the initial datum in (1.5) satisfies

$$
\delta^{2} \varphi_{i}-\varphi_{i}^{-p_{i}} \leqslant-A \varphi_{i}^{-p_{-}}, \quad 0 \leqslant i \leqslant I .
$$

Then the solution $U_{h}(t)$ of $(1.4)-(1.5)$ is quenched in a finite time $T_{b}^{h}$ and we have the following estimate:

$$
T_{b}^{h} \leqslant \frac{1}{A} \frac{\left(\varphi_{h \min }\right)^{p_{-}+1}}{\left(p_{-}+1\right)}
$$

where $\varphi_{h \min }=\min _{0 \leqslant i \leqslant I} \varphi_{i}$ and $0<p_{-}=\min _{0 \leqslant i \leqslant I} p_{i}$ 
Proof. Let $\left(0, T_{b}^{h}\right)$ be the maximal time interval of existence of the solution $U_{h}(t)$. Our aim is to show that $T_{b}^{h}$ is finite and obeys the above inequality. For this fact, let us introduce a vector $J_{h}$ such that

$$
J=\frac{d U}{d t}+A U^{-p_{-}}
$$

A straightforward calculation gives

$$
\frac{d J}{d t}-\delta^{2} J=\frac{d}{d t}\left(\frac{d U}{d t}-\delta^{2} U\right)-A p_{-} U^{-p_{-}-1} \frac{d U}{d t}-A \delta^{2} U^{-p_{-}} .
$$

From Lemma 2.3, we have $\delta^{2} U^{-p_{-}} \geqslant-p_{-} U^{-p_{-}-1} \delta^{2} U$, which implies that

$$
\frac{d J}{d t}-\delta^{2} J \leqslant \frac{d}{d t}\left(\frac{d U}{d t}-\delta^{2} U\right)-A p_{-} U^{-p_{-}-1}\left(\frac{d U}{d t}-\delta^{2} U\right) .
$$

Use (1.4) and the fact that $p_{-} \leqslant p_{i}$ for $0 \leqslant i \leqslant I$ to obtain

$$
\frac{d J}{d t}-\delta^{2} J \leqslant p_{i} U^{-p_{i}-1} \frac{d U}{d t}+A p_{i} U^{-p_{-}-p_{i}-1} .
$$

Taking into account the expression $J_{h}(t)$, we discover that

$$
\frac{d J}{d t}-\delta^{2} J \leqslant p_{i} U^{-p_{i}-1} J
$$

Obviously, the hypotheses on the initial datum in $(2.3)$ ensure that $J_{h}(0) \leqslant 0$. It follows from Lemma 2.1 that $J_{h}(t) \leqslant 0$, which implies that $\frac{d U}{d t} \leqslant-A U^{-p_{-}}$. This estimation may be rewritten as follows

$$
U^{p_{-}} d U \leqslant-A d t
$$

Integrating this inequality with respect to $\left(t, T_{b}^{h}\right)$, we find that

$$
T_{b}^{h}-t \leqslant \frac{1}{A} \frac{(U(t))^{p_{-}+1}}{\left(p_{-}+1\right)} .
$$

Using the fact that $\varphi_{h \text { min }}=U_{i_{0}}$ for a certain $i_{0} \in\{0, \ldots, I\}$ and taking $t=0$ in (2.4), we obtain

$$
T_{b}^{h} \leqslant \frac{1}{A} \frac{\varphi_{h \min }^{p_{-}+1}}{\left(p_{-}+1\right)} .
$$

The fact that the quantity on the right hand side of the above inequality is finite completes the rest of the proof.

Remark 2.1. It is clear that the exponent $p_{-}$depends on $h$. In order to obtain the convergence of the quenching time, we need to get an estimate which does not depend on $h$. It follows from inequalities (2.4) that

$$
T_{b}^{h}-t_{0} \leqslant \frac{1}{A} \frac{\left(U_{h \min }\left(t_{0}\right)^{p_{-}+1}\right)}{\left(p_{-}+1\right)} \quad \text { if } \quad 0<t_{0}<T_{b}^{h}
$$

Since $p_{-}$approaches $p_{*}$, when $h$ tends to zero, the expression $\frac{1}{A} \frac{\left(U_{h \min }\left(t_{0}\right)^{p_{-}+1}\right)}{\left(p_{-}+1\right)}$ approaches $\frac{1}{A} \frac{\left(U_{h \min }\left(t_{0}\right)^{p_{*}+1}\right)}{\left(p_{*}+1\right)}$ which is finite.

Theorem 2.2. Let $U_{h}(t)$ be the solution of (1.4)-(1.5). Then we have

$$
T_{b}^{h} \geqslant \frac{\varphi_{h \min }^{p_{-}+1}}{\left(p_{-}+1\right)} \quad \text { and } \quad U_{h \min }(t) \leqslant\left(p_{-}+1\right)^{\frac{1}{p_{-}+1}}\left(T_{b}^{h}-t\right)^{\frac{1}{p_{-}+1}} \quad \text { for } \quad t \in\left(0, T_{b}^{h}\right) .
$$

Proof. See [22] and use the fact that $p_{-} \leqslant p_{i}$ for $0 \leqslant i \leqslant I$ to complete the proof. 


\section{Convergence of the semidiscrete quenching time}

We now turn our attention to the convergence of the semidiscrete quenching time. To prove this result, we first show that for each fixed time interval $[0, T]$, where the solution $u$ of $(1.1)-$ (1.3) is defined, the solution $U_{h}(t)$ of (1.4)-(1.5) approximates $u$ when the mesh parameter $h$ goes to zero by the following theorem.

Theorem 3.1. Assume that (1.1)-(1.3) has a solution $u \in C^{3,1}([0,1] \times[0, T])$ such that $\min _{t \in[0, T]} u_{\min }(t)=\alpha>0$ and the initial condition in (1.5) and the exponent in (1.4) satisfy

$$
\left\|\varphi_{h}-u_{h}(0)\right\|_{\infty}=o(1) \quad \text { and } \quad\left\|p_{h}-p\right\|_{\infty}=o(1) \quad \text { as } \quad h \rightarrow 0
$$

where $u_{h}(t)=\left(u\left(x_{0}, t\right), \ldots, u\left(x_{I}, t\right)\right)^{\top}$. Then, for $h$ sufficiently small, problem (1.4)-(1.5) has a unique solution $U_{h} \in C^{1}\left([0, T], \mathbb{R}^{I+1}\right)$ such that

$$
\max _{0 \leqslant t \leqslant T}\left\|U_{h}(t)-u_{h}(t)\right\|_{\infty}=O\left(\left\|\varphi_{h}-u_{h}(0)\right\|_{\infty}+\left\|p_{h}-p\right\|_{\infty}+h\right) \quad \text { as } \quad h \rightarrow 0 .
$$

Proof. Since $u \in C^{3,1}$, there exist positive constants $K$ and $M$ such that

$$
\frac{\left\|u_{x x x}\right\|_{\infty}}{3} \leqslant K, \quad p_{+}\left(\frac{\alpha}{2}\right)^{-p_{-}-1} \leqslant M .
$$

Problem (1.4)-(1.5) has for each $h$, a unique solution $U_{h} \in C^{1}\left(\left[0, T_{b}^{h}\right), \mathbb{R}^{I+1}\right)$. Let $t(h)$ be the largest value of $t>0$ such that

$$
\left\|U_{h}(t)-u_{h}(t)\right\|_{\infty}<\frac{\alpha}{2} \quad \text { for } \quad t \in(0, t(h)) .
$$

Relation (3.1) implies that $t(h)>0$ for $h$ sufficiently small. Let $t^{*}(h)=\min \{t(h), T\}$. By the triangle inequality, we obtain

$$
U_{h \min }(t) \geqslant u_{h \min }(t)-\left\|U_{h}(t)-u_{h}(t)\right\|_{\infty} \quad \text { for } \quad t \in\left(0, t^{*}(h)\right),
$$

which implies that

$$
U_{h \min }(t) \geqslant \alpha-\frac{\alpha}{2}=\frac{\alpha}{2} \quad \text { for } \quad t \in\left(0, t^{*}(h)\right) .
$$

Applying Taylor's expansion, we have for $t \in\left(0, t^{*}(h)\right)$,

$$
\begin{gathered}
u_{x x}\left(x_{i}, t\right)=\delta^{2} u\left(x_{i}, t\right)-\frac{h}{3} u_{x x x}\left(\bar{x}_{i}, t\right), \quad 0 \leqslant i \leqslant I-1, \\
u_{x x}\left(x_{I}, t\right)=\delta^{2} u\left(x_{I}, t\right)+\frac{h}{3} u_{x x x}\left(\bar{x}_{I}, t\right) .
\end{gathered}
$$

Let $e_{h}(t)=U_{h}(t)-u_{h}(t)$ be the error of discretization. Since $u \in C^{3,1}$, from the mean value theorem, we have for $t \in\left(0, t^{*}(h)\right)$,

$$
\begin{gathered}
\frac{d e_{i}(t)}{d t}-\delta^{2} e_{i}(t)=-p_{i} \xi_{i}^{-p_{i}-1} e_{i}(t)+\ln \left(u\left(x_{i}, t\right)\right) u\left(x_{i}, t\right)^{-s_{i}}\left(p_{i}-p\left(x_{i}\right)\right)-\frac{h}{3} u_{x x x}\left(\widetilde{x}_{i}, t\right), \quad 0 \leqslant i \leqslant I-1, \\
\frac{d e_{I}(t)}{d t}-\delta^{2} e_{I}(t)=-p_{I} \xi_{I}^{-p_{I}-1} e_{I}(t)+\ln \left(u\left(x_{I}, t\right)\right) u\left(x_{I}, t\right)^{-s_{I}}\left(p_{I}-p\left(x_{I}\right)\right)+\frac{h}{3} u_{x x x}\left(\widetilde{x}_{I}, t\right),
\end{gathered}
$$


where $\xi_{i}$ is an intermediate value between $U_{i}(t)$ and $u\left(x_{i}, t\right)$ and $s_{i}$ is the one between $p\left(x_{i}\right)$ and $p_{i}$. According to (3.3), we find that

$$
p_{i} \xi_{i}^{-p_{i}-1} \leqslant M \quad \text { and } \quad\left|\ln \left(u\left(x_{i}, t\right)\right)\left(u\left(x_{i}, t\right)\right)^{-s_{i}}\right| \leqslant M, \quad 0 \leqslant i \leqslant I,
$$

where $M=\max \left\{p_{+}(\alpha / 2)^{-p_{-}-1},(\alpha / 2)^{-p_{-}}|\ln (\alpha / 2)|\right\}$. Using (3.3), (3.5), and the fact that $u_{x x x}(x, t)$ is bounded, we find that there exists a positive constant $M$ such that

$$
\frac{d e(t)}{d t}-\delta^{2} e(t) \leqslant M|e(t)|+M\left|p_{i}-p\right|+M h, \quad t \in\left(0, t^{*}(h)\right) .
$$

Introduce a vector $Z_{h}(t)$ such that

$$
Z(t)=e^{(M+1) t}\left(\left\|\varphi_{h}-u_{h}(0)\right\|_{\infty}+M\left\|p_{h}-p\right\|_{\infty}+M h\right), \quad t \in\left(0, t^{*}(h)\right) .
$$

A straightforward calculation reveals that

$$
\frac{d Z(t)}{d t}-\delta^{2} Z(t)>M|Z(t)|+M\left\|p_{h}-p\right\|_{\infty}+M h, \quad Z(0)>e(0) \quad t \in\left(0, t^{*}(h)\right) .
$$

It follows from Lemma 2.2 that $Z_{h}(t)>e_{h}(t)$ for $t \in\left(0, t^{*}(h)\right)$. In the same way, we also prove that $Z_{h}(t)>-e_{h}(t)$ for $t \in\left(0, t^{*}(h)\right)$, which implies that

$$
\left\|U_{h}(t)-u_{h}(t)\right\|_{\infty} \leqslant e^{(M+1) t}\left(\left\|\varphi_{h}-u_{h}(0)\right\|_{\infty}+M\left\|p_{h}-p\right\|_{\infty}+M h\right), \quad t \in\left(0, t^{*}(h)\right) .
$$

Let us show that $t^{*}(h)=T$. Suppose that $T>t(h)$. From (3.4) we obtain

$$
\frac{\alpha}{2} \leqslant\left\|U_{h}(t(h))-u_{h}(t(h))\right\|_{\infty} \leqslant e^{(M+1) T}\left(\left\|\varphi_{h}-u_{h}(0)\right\|_{\infty}+M\left\|p_{h}-p\right\|_{\infty}+M h\right) .
$$

Since the term on the right hand side of the above inequality tends to zero as $h$ goes to zero, we deduce that $\alpha / 2 \leqslant 0$, which is impossible. Consequently $t^{*}(h)=T$, and the proof is completed.

Now, we are in a position to prove the main theorem of this section.

Theorem 3.2. Suppose that problem (1.1)-(1.3) has a solution $u$ which is quenched in a finite time $T_{b}$ such that $u \in C^{3,1}\left([0,1] \times\left[0, T_{b}\right)\right)$ and the initial datum in (1.5) satisfies

$$
\left\|\varphi_{h}-u_{h}(0)\right\|_{\infty}=o(1) \quad \text { and } \quad\left\|p_{h}-p\right\|_{\infty}=o(1) \quad \text { as } \quad h \rightarrow 0 .
$$

Under the assumptions of Theorem 2.1, problem (1.4)-(1.5) has a solution $U_{h}(t)$ which is quenched in a finite time $T_{b}^{h}$ and

$$
\lim _{h \rightarrow 0} T_{b}^{h}=T_{b} .
$$

Proof. Let $\varepsilon>0$. There exists a positive constant $\rho$ such that

$$
\frac{1}{A} \frac{x^{p_{*}+1}}{\left(p_{*}+1\right)} \leqslant \frac{\varepsilon}{2}<\infty \quad \text { for } \quad 0 \leqslant x \leqslant \rho .
$$

Since $u$ is quenched at the time $T_{b}$, then there exists $T_{1}$ such that $\left|T_{1}-T_{b}\right| \leqslant \varepsilon / 2$ and $0<u_{\min }(t) \leqslant \rho / 2$ for $t \in\left[T_{1}, T_{b}\right)$. Letting $T_{2}=\left(T_{1}+T_{b}\right) / 2$, we see that $0<u_{\min }(t)$ for $t \in\left[0, T_{1}\right]$. It follows from Theorem 3.1 that problem $(1.4)-(1.5)$ has a solution $U_{h}(t)$ which obeys $\sup _{t \in\left[0, T_{2}\right]}\left|U_{h}(t)-u_{h}(t)\right|_{\infty} \leqslant \rho / 2$. Applying the triangle inequality, we get $U_{h \min }(t) \leqslant$ $u_{h \text { min }}(t)+\left\|U_{h}(t)-u_{h}(t)\right\|_{\infty}$, which leads to $U_{h \text { min }}(t) \leqslant \rho$ for $t \in\left[T_{1}, T_{2}\right]$. From Theorem 2.1, $U_{h}(t)$ is quenched at the time $T_{b}^{h}$. We deduce from Remark 2.1 and (3.8) that

$$
\left|T_{b}^{h}-T_{b}\right| \leqslant\left|T_{b}^{h}-T_{2}\right|+\left|T_{2}-T_{b}\right| \leqslant \frac{\varepsilon}{2}+\frac{1}{A} \frac{\left(U_{h \min }\left(T_{2}\right)\right)^{p_{*}+1}}{\left(p_{*}+1\right)} \leqslant \varepsilon,
$$

and we have the desired result. 


\section{Quenching of solutions}

In this section, we study the phenomenon of quenching using an explicit scheme of (1.1)-(1.3). Under some assumptions, we show that the solution of the discrete problem is quenched in a finite time and estimate its numerical quenching time. We start by constructing a discrete explicit scheme as follows. Approximate the solution $u(x, t)$ of problem (1.1)-(1.3) by the solution $U_{h}^{(n)}=\left(U_{0}^{(n)}, U_{1}^{(n)}, \ldots, U_{I}^{(n)}\right)^{\top}$ of the following explicit scheme:

$$
\begin{gathered}
\delta_{t} U_{i}^{(n)}=\delta^{2} U_{i}^{(n)}-\left(U_{i}^{(n)}\right)^{-p_{i}}, \quad 0 \leqslant i \leqslant I, \\
U_{i}^{(0)}=\varphi_{i}>0, \quad 0 \leqslant i \leqslant I,
\end{gathered}
$$

where $n \geqslant 0$,

$$
\delta_{t} U_{i}^{(n)}=\frac{U_{i}^{(n+1)}-U_{i}^{(n)}}{\Delta t_{n}} .
$$

To let the discrete solution reproduce the properties of the continuous one when the time $t$ approaches the quenching time $T_{h}^{\Delta t}$, we need to adapt the size of the time step. We choose

$$
\Delta t_{n}=\min \left\{\frac{(1-\tau) h^{2}}{3}, \tau\left(U_{h \min }^{(n)}\right)^{1+p_{-}}\right\}, \quad 0<\tau<1 .
$$

Let us notice that the restriction on the time step ensures the positivity of the discrete solution (see $[22]$ ).

In this second part, we shall use the following notational conventions:

$$
U=U_{i}^{(n)}, \quad \delta^{2} U=\delta^{2} U_{i}^{(n)} \quad \text { and } \quad \delta_{t} U=\delta_{t} U_{i}^{(n)} .
$$

The following lemma is a discrete version of the maximum principle.

Lemma 4.1. Let $a^{(n)}$ and $V_{h}^{(n)}$ be two sequences such that $a^{(n)}$ is bounded and

$$
\delta_{t} V-\delta^{2} V+a V \geqslant 0, \quad V^{(0)} \geqslant 0 .
$$

Then $V \geqslant 0$ if $\Delta t_{n} \leqslant h^{2} / 2$.

Proof. See [22].

A direct consequence of the above result is the following comparison lemma. Its proof is straightforward.

Lemma 4.2. Suppose that $a^{(n)}, b^{(n)}, V_{h}^{(n)}$ and let $W_{h}^{(n)}$ be four sequences such that $a^{(n)}$ is bounded and

$$
\delta_{t} V-\delta^{2} V+a V+b \leqslant \delta_{t} W-\delta^{2} W+a W, \quad V^{(0)} \leqslant W^{(0)} .
$$

Then $V \leqslant W$ if $\Delta t_{n} \leqslant h^{2} / 2$.

Now, let us end this section with some properties of the approximate solution $U$.

Lemma 4.3. Let $U^{(n)} \in \mathbb{R}^{I+1}$ be such that $U^{(n)}>0$ for $n \geqslant 0$, and let $\gamma \in \mathbb{R} \backslash[0,1]$. Then we have

$$
\text { (i) } \delta_{t} U^{\gamma} \geqslant \gamma U^{\gamma-1} \delta_{t} U
$$

And if the initial datum in (4.2) satisfies $\varphi \leqslant 1$ and $\delta_{t} U^{(0)} \leqslant 0$, then we have

$$
\text { (ii) } \quad U^{(n+1)} \leqslant U^{(n)} \leqslant 1, \quad n \geqslant 0,
$$

where $U^{(n)}$ is the solution of (4.1)-(4.2). 
Proof. To prove $(i)$, use the Taylor expansion of $\left(U^{(n+1)}\right)^{\gamma}$ and we have the desired result.

To prove $(i i)$, introduce the function $w=\delta_{t} U$. A straightforward computation yields

$$
\delta_{t} w-\delta^{2} w=\delta_{t}\left(\delta_{t} U-\delta^{2} U\right) .
$$

Using (4.1), we arrive at $\delta_{t} w-\delta^{2} w=-\delta_{t} U^{-p_{i}}$. It follows from $(i)$ that

$$
\delta_{t} w-\delta^{2} w \leqslant p_{i} U^{-p_{i}-1} w .
$$

From Lemma 4.1, we deduce that $w_{h} \leqslant 0$, which implies that

$$
U^{(n+1)} \leqslant U^{(n)} \leqslant \varphi \leqslant 1, \quad n \geqslant 0
$$

and we have the desired result.

To handle the phenomenon of quenching for discrete equations, we need the following definition.

Definition 4.1. We say that the solution $U_{h}^{(n)}$ of $(4.1)-(4.2)$ is quenched in a finite time if $U_{h \min }^{(n)}>0$ for $n \geqslant 0$, but

$$
\lim _{n \rightarrow \infty} U_{h \min }^{(n)}=0 \quad \text { and } \quad T_{h}^{\Delta t}=\lim _{n \rightarrow \infty} \sum_{i=0}^{n-1} \Delta t_{i}<\infty .
$$

The number $T_{h}^{\Delta t}$ is called the numerical quenching time of $U_{h}^{(n)}$.

The following theorem reveals that the discrete solution $U_{h}^{(n)}$ of $(4.1)-(4.2)$ is quenched in a finite time under some hypotheses.

Theorem 4.1. Let $U_{h}^{(n)}$ be the solution of (4.1)-(4.2). Suppose that there exists a constant $A \in(0,1]$ such that the initial datum in (4.2) satisfies $\varphi \leqslant 1$ and

$$
\delta^{2} \varphi_{i}-\varphi_{i}^{-p_{i}} \leqslant-A \varphi_{i}^{-p_{-}}, \quad 0 \leqslant i \leqslant I .
$$

Then $U_{h}^{(n)}$ is quenched in a finite time $T_{h}^{\Delta t}$ which satisfies the following estimate:

$$
T_{h}^{\Delta t} \leqslant \frac{\tau \varphi_{h \min }^{p_{-}+1}}{1-\left(1-\tau^{\prime}\right)^{p_{-}+1}}, \quad \text { where } \quad \tau^{\prime}=A \min \left\{\frac{(1-\tau) h^{2}}{3} \varphi_{h \min }^{-p_{-}}, \tau\right\} .
$$

Proof. Introduce the vector $J_{h}$ defined as follows:

$$
J=\delta_{t} U+A U^{-p_{-}} .
$$

A straightforward computation yields

$$
\delta_{t} J-\delta^{2} J=\delta_{t}\left(\delta_{t} U-\delta^{2} U\right)+A \delta_{t} U^{-p_{-}}-A \delta^{2} U^{-p_{-}} .
$$

Using (4.1), we arrive at

$$
\delta_{t} J-\delta^{2} J=-\delta_{t} U^{-p_{i}}+A \delta_{t} U^{-p_{-}}-A \delta^{2} U^{-p_{-}} .
$$

By direct calculation and using $(i)$ of Lemma 4.3 we have

$$
\delta_{t} U^{-p_{i}}=\delta_{t}\left(U^{-p_{-}}\right)^{p_{i} / p_{-}} \geqslant \frac{p_{i}}{p_{-}}\left(U^{-p_{-}}\right)^{p_{i} / p_{-}-1} \delta_{t} U^{-p_{-}} .
$$


From (ii) of Lemma 4.3, we have $U^{-p_{i}+p_{-}} \geqslant 1$, which leads to

$$
\delta_{t} J-\delta^{2} J \leqslant-\left(\frac{p_{i}}{p_{-}} U^{-p_{i}+p_{-}}-A\right) \delta_{t} U^{-p_{-}}-A \delta^{2} U^{-p_{-}} .
$$

It follows from Lemmas 2.3 and 4.3 that

$$
\delta_{t} J-\delta^{2} J \leqslant\left(\frac{p_{i}}{p_{-}} U^{-p_{i}+p_{-}}-A\right) p_{-} U^{-p_{-}-1} \delta_{t} U+A p_{-} U^{-p_{-}-1} \delta^{2} U,
$$

which implies that

$$
\delta_{t} J-\delta^{2} J \leqslant p_{i} U^{-p_{i}-1} \delta_{t} U-A p_{-} U^{-p_{-}-1}\left(\delta_{t} U-\delta^{2} U\right) .
$$

Using (4.1) and the fact that $p_{i} \geqslant p_{-}$for $0 \leqslant i \leqslant I$, we have

$$
\delta_{t} J-\delta^{2} J \leqslant p_{-} U^{-p_{i}-1} \delta_{t} U+A p_{-} U^{-p_{-}-p_{i}-1},
$$

which leads to

$$
\delta_{t} J-\delta^{2} J \leqslant p_{-} U^{-p_{i}-1} J
$$

Obviously from $(4.4)$, we see that $J_{h}^{(0)} \leqslant 0$. It follows from Lemma 4.1 that $J_{h} \leqslant 0$, which implies that

$$
\delta_{t} U+A U^{-p_{-}} \leqslant 0
$$

Consequently, we get

$$
U_{h \min }^{(n+1)} \leqslant U_{h \min }^{(n)}-A \Delta t_{n}\left(U_{h \min }^{(n)}\right)^{-p_{-}} .
$$

This inequality shows that the sequence $U_{h \min }^{(n)}$ is decreasing. By induction we obtain $U_{h \min }^{(n+1)} \leqslant$ $U_{h \min }^{(n)} \leqslant \varphi_{h \text { min }}$. Thus, the following holds:

$$
A \Delta t_{n}\left(U_{h \min }^{(n)}\right)^{-p_{-}-1} \geqslant A \min \left\{\frac{(1-\tau) h^{2}}{3} \varphi_{h \min }^{-p_{-}-1}, \tau\right\}=\tau^{\prime} .
$$

Consequently, we get

$$
U_{h \min }^{(n+1)} \leqslant U_{h \min }^{(n)}\left(1-\tau^{\prime}\right), \quad n \geqslant 0,
$$

and by iteration we arrive at

$$
U_{h \min }^{(n)} \leqslant U_{h \min }^{(0)}\left(1-\tau^{\prime}\right)^{n}=\varphi_{h \min }\left(1-\tau^{\prime}\right)^{n}, \quad n \geqslant 0 .
$$

Since the term on the right hand side of the above equality goes to zero as $n$ approaches infinity, we conclude that $U_{h \min }^{(n)}$ tends to zero as $n$ approaches infinity.

Due to (4.7) and the restriction $\Delta t_{n}$ the numerical blow-up time can be estimated as follows:

$$
T_{h}^{\Delta t}=\sum_{n=0}^{+\infty} \Delta t_{n} \leqslant \tau \sum_{n=0}^{+\infty} \varphi_{h \min }^{p_{-}+1}\left[\left(1-\tau^{\prime}\right)^{p_{-}+1}\right]^{n} .
$$

Use the fact that the series on the right hand side of the above inequality converges toward $\tau \varphi_{h \min }^{p_{-}+1} /\left(1-\left(1-\tau^{\prime}\right)^{p_{-}+1}\right)$ to complete the rest of the proof. 
Remark 4.1. We deduce from (4.6) that

$$
U_{h \min }^{(n)} \leqslant U_{h \min }(q)\left(1-\tau^{\prime}\right)^{n-q} \text { for } n \geqslant q,
$$

and we have

$$
T_{h}^{\Delta t}-t_{q}=\sum_{n=q}^{+\infty} \Delta t_{n} \leqslant \sum_{n=q}^{+\infty} \tau\left(U_{h \min }^{(q)}\right)^{p_{-}+1}\left[\left(1-\tau^{\prime}\right)^{p_{-}+1}\right]^{n-q}
$$

It follows that

$$
T_{h}^{\Delta t}-t_{q} \leqslant \frac{\tau\left(U_{h \min }^{(q)}\right)^{p_{-+1}}}{1-\left(1-\tau^{\prime}\right)^{p_{-}+1}} \simeq \frac{\tau\left(U_{h \min }^{(q)}\right)^{p_{*}+1}}{1-\left(1-\tau^{\prime}\right)^{p_{*}+1}}
$$

when $h$ tends to zero. From $\tau^{\prime}=A \min \left\{(1-\tau) h^{2} \varphi_{h \min }^{-p_{-}^{-1}} / 3, \tau\right\}$, if we take $\tau=h^{2}$, we obtain

$$
\frac{\tau^{\prime}}{\tau}=A \min \left\{\frac{\left(1-h^{2}\right) \varphi_{h \min }^{-p_{-}-1}}{3}, 1\right\} \geqslant A \min \left\{\frac{\varphi_{h \min }^{-p_{-}-1}}{4}, 1\right\} \simeq A \min \left\{\frac{\varphi_{h \min }^{-p_{*}-1}}{4}, 1\right\}
$$

when $h$ tends to zero. Therefore, there exist constants $c_{0}$ and $c_{1}$ such that $0 \leqslant c_{0} \leqslant \tau / \tau^{\prime} \leqslant c_{1}$ and $\tau /\left(1-\left(1-\tau^{\prime}\right)^{p_{*}+1}\right)=O(1)$, for the choice of $\tau=h^{2}$.

We will use $\tau=h^{2}$ in the following.

\section{Convergence of the numerical blow-up time}

In this section, under some conditions, we show that the solution of the discrete problem is quenched in a finite time and its numerical quenching time goes to the real one when the mesh size tends to zero. First, let us prove the convergence of our scheme by the following.

Theorem 5.1. Suppose that problem (1.1)-(1.3) has a solution $u \in C^{3,2}([0,1] \times[0, T])$ such that $\min _{t \in[0, T]} u_{\min }(t)=\alpha>0$ and the initial datum in (4.2) verifies

$$
\left\|\varphi_{h}-u_{h}(0)\right\|_{\infty}=o(1) \quad \text { and } \quad\left\|p_{h}-p\right\|_{\infty}=o(1) \quad \text { as } \quad h \rightarrow 0 .
$$

Then problem (4.1)-(4.2) has a solution $U_{h}^{(n)}$ for $h$ sufficiently small, $0 \leqslant n \leqslant Q$ and the following estimate holds:

$$
\max _{0 \leqslant n \leqslant Q}\left\|U_{h}^{(n)}-u_{h}\left(t_{n}\right)\right\|_{\infty}=O\left(\left\|\varphi_{h}-u_{h}(0)\right\|_{\infty}+M\left\|p_{h}-p\right\|_{\infty}+h\right) \quad \text { as } \quad h \rightarrow 0,
$$

where $Q$ is such that $\sum_{n=0}^{Q-1} \Delta t_{n} \leqslant T$ and $t_{n}=\sum_{j=0}^{n-1} \Delta t_{j}$.

Proof. Since $u \in C^{3,1}$, there exist positive constants $K$ and $M$ such that

$$
\frac{\left\|u_{x x x}\right\|_{\infty}}{3} \leqslant K, \quad p_{+}\left(\frac{\alpha}{2}\right)^{-p_{-}-1} \leqslant M
$$

For each $h$, problem (4.1)-(4.2) has a solution $U_{h}^{(n)}$. Let $N \leqslant Q$ be the largest value of $n$ such that

$$
\left\|U_{h}^{(n)}-u_{h}\left(t_{n}\right)\right\|_{\infty}<\frac{\alpha}{2} \quad \text { for } \quad n<N
$$


We deduce from (5.1) that $N \geqslant 1$. Applying the triangle inequality, we have

$$
U_{h \min }^{(n)} \geqslant u_{h \min }\left(t_{n}\right)-\left\|U_{h}^{(n)}-u_{h}\left(t_{n}\right)\right\|_{\infty} \text { for } n<N,
$$

which implies that

$$
U_{h \min }^{(n)} \geqslant \alpha-\frac{\alpha}{2} \quad \text { for } \quad n<N .
$$

Applying Taylor's expansion, we have for $n<N$

$$
\delta_{t} u\left(x_{i}, t_{n}\right)=u_{t}\left(x_{i}, t_{n}\right)+\frac{\Delta t_{n}}{2} u_{t t}\left(x_{i}, \tilde{t_{n}}\right), \quad 0 \leqslant i \leqslant I .
$$

Introduce the error of discretization $e_{h}^{(n)}=U_{h}^{(n)}-u_{h}\left(t_{n}\right)$. As in the proof of Theorem 3.1, from the mean value theorem, we get for $n<N$

$$
\begin{gathered}
\delta_{t} e-\delta^{2} e=-p_{i} \xi^{-p_{i}-1} e+\left(u\left(x_{i}, t_{n}\right)\right)^{-s_{i}} \ln \left(u\left(x_{i}, t_{n}\right)\right)\left(p_{i}-p\left(x_{i}\right)\right)- \\
\frac{h}{3} u_{x x x}\left(\widetilde{x}_{i}, t_{n}\right)-\frac{\Delta t_{n}}{2} u_{t t}\left(x_{i}, \widetilde{t}_{n}\right), \quad 0 \leqslant i \leqslant I-1, \\
\delta_{t} e_{I}^{(n)}-\delta^{2} e_{I}^{(n)}=-p_{I}\left(\xi_{I}^{(n)}\right)^{-p_{I}-1} e_{I}^{(n)}+\left(u\left(x_{I}, t_{n}\right)\right)^{-s_{I}} \ln \left(u\left(x_{I}, t_{n}\right)\right)\left(p_{I}-p\left(x_{I}\right)\right)+ \\
\frac{h}{3} u_{x x x}\left(\widetilde{x}_{I}, t_{n}\right)-\frac{\Delta t_{n}}{2} u_{t t}\left(x_{I}, \widetilde{t}_{n}\right),
\end{gathered}
$$

where $\xi$ is an intermediate value between $U$ and $u$. Since $\left|u\left(x_{i}, t_{n}\right)^{-s_{i}} \ln \left(u\left(x_{i}, t_{n}\right)\right)\right| \leqslant M$, $u_{x x x}(x, t), u_{t t}(x, t)$ are bounded, $\Delta t_{n}=O\left(h^{2}\right)$ and using (5.2) and (5.5), we obtain that there exists a positive constant $M$ such that

$$
\delta_{t} e-\delta^{2} e \leqslant M e+M\left(p_{i}-p\right)+M h .
$$

Let the vector $V_{h}^{(n)}$ be defined as follows:

$$
V=e^{(M+1) t_{n}}\left(\left\|\varphi_{h}-u_{h}(0)\right\|_{\infty}+M\left\|p_{h}-p\right\|_{\infty}+M h\right) .
$$

Direct calculation yealds that

$$
\delta_{t} V-\delta^{2} V>M V+M\left\|p_{h}-p\right\|_{\infty}+M h, \quad V^{(0)}>e^{(0)} .
$$

From Comparison Lemma 4.2 we deduce that $V_{h}^{(n)} \geqslant e_{h}^{(n)}$. Likewise, we also prove that $V_{h}^{(n)} \geqslant-e_{h}^{(n)}$, which implies that

$$
\left\|U_{h}^{(n)}-u_{h}\left(t_{n}\right)\right\|_{\infty} \leqslant e^{(M+1) t_{n}}\left(\left\|\varphi_{h}-u_{h}(0)\right\|_{\infty}+M\left\|p_{h}-p\right\|_{\infty}+M h\right) .
$$

Let us show that $N=Q$. Suppose that $N<Q$. If we replace $n$ by $N$ in (5.8) and use (5.3), then we find that

$$
\frac{\alpha}{2} \leqslant\left\|U_{h}^{(N)}-u_{h}\left(t_{N}\right)\right\|_{\infty} \leqslant e^{(M+1) T}\left(\left\|\varphi_{h}-u_{h}(0)\right\|_{\infty}+M\left\|p_{h}-p\right\|_{\infty}+M h\right) .
$$

Since the term on the right hand side of the second inequality goes to zero as $h$ goes to zero, we deduce that $\alpha / 2 \leqslant 0$, which is a contradiction and the proof is completed.

Now, we are in a position to state the main theorem of this section. 
Theorem 5.2. Suppose that problem (1.1)-(1.3) has a solution u which is quenched in a finite time $T_{b}$ and $u \in C^{3,2}\left([0,1] \times\left[0, T_{b}\right)\right)$. Assume that the initial datum in (4.2) satisfies

$$
\left\|\varphi_{h}-u_{h}(0)\right\|_{\infty}=o(1) \quad \text { and } \quad\left\|p_{h}-p\right\|_{\infty}=o(1) \quad \text { as } \quad h \rightarrow 0 .
$$

Under the assumption of Theorem 4.1, problem (4.1)-(4.2) has a solution $U_{h}^{(n)}$ which is quenched in a finite time $T_{h}^{\Delta t}$ and the following relation holds:

$$
\lim _{h \rightarrow 0} T_{h}^{\Delta t}=T_{b} .
$$

Proof. Letting $\varepsilon>0$, there exists a constant $\alpha>0$ such that

$$
\frac{\tau x^{p_{*}+1}}{1-\left(1-\tau^{\prime}\right)^{p_{*}+1}}<\frac{\varepsilon}{2}<\infty \text { for } 0 \leqslant x \leqslant \alpha .
$$

Since $u$ is quenched at the time $T_{b}$, there exists $T_{1}$ such that $\left|T_{1}-T_{b}\right|<\varepsilon / 2$ and $0<$ $u_{h \min }(t)<\alpha / 2$ for $t \in\left[T_{1}, T_{b}\right)$. Let $T_{2}=\left(T_{1}+T_{b}\right) / 2$ and let $q$ be a positive integer such that $t_{q}=\sum_{n=0}^{q-1} \Delta t_{n} \in\left[T_{1}, T_{2}\right]$ for $h$ small enough. It follows from Theorem 5.1 that problem (4.1)-(4.2) has a solution $U_{h}^{(n)}$ obeying $\left\|U_{h}^{(n)}-u_{h}\left(t_{n}\right)\right\|_{\infty}<\alpha / 2$ for $n \geqslant q$, which implies that

$$
U_{h \min }^{(q)} \leqslant u_{h \min }\left(t_{q}\right)+\left\|U_{h}^{(q)}-u_{h}\left(t_{q}\right)\right\|_{\infty}<\frac{\alpha}{2}+\frac{\alpha}{2}=\alpha .
$$

From Theorem 4.1, $U_{h}^{(n)}$ is quenched at the time $T_{h}^{\Delta t}$. It follows from Remark 4.1 and (5.10) that

$$
\left|T_{h}^{\Delta t}-t_{q}\right| \leqslant \frac{\tau\left(U_{h \min }^{(q)}\right)^{p_{*}+1}}{1-\left(1-\tau^{\prime}\right)^{p_{*}+1}}<\frac{\varepsilon}{2}
$$

because $U_{h \min }^{(q)}<\alpha$. We deduce that $\left|T-T_{h}^{\Delta t}\right| \leqslant\left|T-t_{q}\right|+\left|t_{q}-T_{h}^{\Delta t}\right| \leqslant \varepsilon / 2+\varepsilon / 2 \leqslant \varepsilon$, which leads us to the result.

\section{Numerical experiments}

In this section, we present some numerical approximations of the quenching time for the solution of problem (1.1)-(1.3) in the case where

$$
p(x)=1+\frac{x}{1+x} \text { and } u_{0}(x)=\frac{2+\cos (\pi x)}{5}
$$

with $0 \leqslant x \leqslant 1$. Firstly, we consider the explicit scheme (4.1)-(4.2). Secondly, we use the following implicit scheme:

$$
\begin{gathered}
\frac{U_{i}^{(n+1)}-U_{i}^{(n)}}{\Delta t_{n}}=\delta^{2} U_{i}^{(n+1)}-\left(U_{i}^{(n)}\right)^{-p_{i}-1} U_{i}^{(n+1)}, \quad 0 \leqslant i \leqslant I, \\
U_{i}^{(0)}=\varphi_{i}, \quad 0 \leqslant i \leqslant I,
\end{gathered}
$$

where $n \geqslant 0, \Delta t_{n}=h^{2}\left(U_{h \min }^{(n)}\right)^{p_{-}+1}$. In both cases,

$$
p_{i}=1+\frac{i h}{1+i h} \text { and } \varphi_{i}=\frac{2+\cos (\pi i h)}{5} .
$$


Let us notice that the restriction on the time step guarantees the positivity of the discrete solution. Also, the existence and the uniqueness of the solution $U_{h}^{(n)}$ is guaranteed with the use of standard methods.

In Tables 6.1 and 6.2, we present the numerical quenching times, the values of $n$, the CPU times, and the orders of the approximations corresponding to meshes of 16, 32, 64, 128, 256. We take for the numerical quenching time $T^{n}=\sum_{j=0}^{n-1} \Delta t_{j}$ which is computed at the first time when $\Delta t_{n}=\left|T^{n+1}-T^{n}\right| \leqslant 10^{-16}$. The order(s) of the method is computed from

$$
s=\frac{\log \left(\left(T_{4 h}-T_{2 h}\right) /\left(T_{2 h}-T_{h}\right)\right)}{\log (2)} .
$$

For numerical values, we take, $U_{i}^{(0)}=\frac{2+\cos (i h \pi)}{5}, p_{i}=1+\frac{i h}{1+i h}$, for $0 \leqslant i \leqslant I$, and $\tau=h^{2}$.

Table 6.1 Numerical quenching times, numbers of iterations, CPU times (seconds) and orders of the approximations obtained with the explicit Euler method

\begin{tabular}{|c|c|c|c|c|}
\hline$I$ & $T^{n}$ & $n$ & $C P U_{t}$ & $s$ \\
\hline 16 & 0.0088515 & 257 & - & - \\
32 & 0.0088713 & 1053 & 1 & - \\
64 & 0.0088784 & 4259 & 4 & 1.48 \\
128 & 0.0088806 & 17129 & 179 & 1.69 \\
256 & 0.0088812 & 68702 & 10873 & 1.87 \\
\hline
\end{tabular}

Table 6.2. Numerical quenching times, numbers of iterations, CPU times (seconds) and orders of the approximations obtained with the first implicit method

\begin{tabular}{|c|c|c|c|c|}
\hline$I$ & $T^{n}$ & $n$ & $C P U_{t}$ & $s$ \\
\hline 16 & 0.0089531 & 270 & - & - \\
32 & 0.0088965 & 1066 & 1 & - \\
64 & 0.0088847 & 4272 & 14 & 2.26 \\
128 & 0.0088821 & 17141 & 451 & 2.18 \\
256 & 0.0088815 & 68709 & 21720 & 2.12 \\
\hline
\end{tabular}

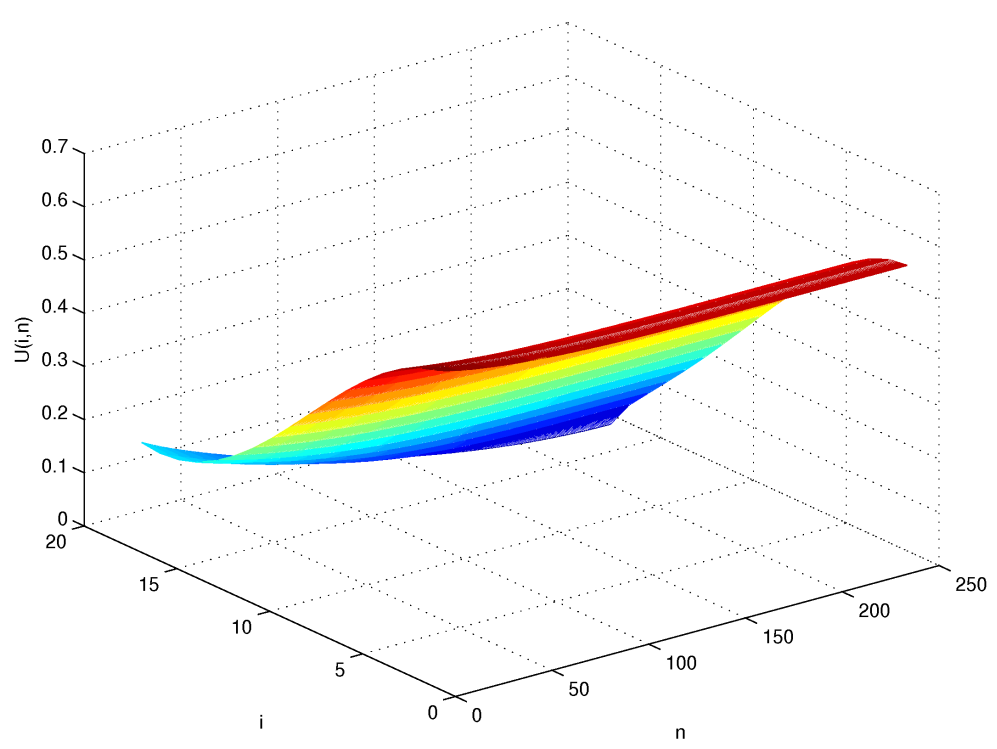

Evolution of the discrete solution of $u(x, t)$

Acknowledgment. We wish to thank the anonymous referee for the thorough reading of the manuscript and several suggestions that helped us improve the presentation of the paper.

\section{References}

1. L. Abia, J. C. López-Marcos, and J. Martinez, On the blow-up time convergence of semidiscretizations of reaction-diffusion equations, Appl. Numer. Math., 26 (1998), pp. 399-414. 
2. A. Acker and W. Walter, The quenching problem for nonlinear parabolic differential equations, Lecture Notes in Math., Springer-Verlag, New York, 564 (1976), pp. 1-12.

3. T. K. Boni, Extinction for discretizations of some semilinear parabolic equations, C. R. Acad. Sci. Paris, Sér. I, 333 (2001), pp. 795-800.

4. T. K. Boni, On quenching of solutions for some semilinear parabolic equations of second order, Bull. Belg. Math. Soc., 7 (2000), pp. 73-95.

5. T. K. Boni and B. Y. Diby, Quenching time of solutions for some nonlinear parabolic equations with Dirichlet boundary condition and a potential, Annales Mathematicae et Informaticae, 35 (2008), pp. 31-42.

6. T. K. Boni and F. K. N'gohisse, Continuity of the quenching time in a semilinear heat equation, An. Univ. Mariae Curie Sklodowska, LXII (2008) pp. 37-48.

7. R. Ferreira, A. de Pablo, and J.L. Vázquez, Classification of blow-up with nonlinear diffusion and localized reaction, J. Differential Equations, 231 (2006), pp. 195-211.

8. R. Ferreira, A. de Pablo, M. Pérez-Llanos and J. D. Rossi, Critical exponent for a parabolic equation with variable reaction, (in print).

9. A. Friedman, Partial differential equations of parabolic type, Printice-Hall, Englewood Cliffs, NJ, 1964.

10. M. Fila, B. Kawohl, and H. A. Levine, Quenching for quasi-linear equations, Comm. Part. Diff. Equat., 17 (1992), pp. 593-614.

11. P. Groisman and J. D. Rossi, Dependance of the blow-up time with respect to parameters and numerical approximations for a parabolic problem, Asympt. Anal., 37 (2004), pp. 79-91.

12. P. Groisman and J.D. Rossi, Asymptotic behaviour for a numerical approximation of a parabolic problem with blowing up solution, J. Compu. Appl. Math., 135 (2001), pp. 135-155.

13. J.Guo, On a quenching problem with Robin boundary condition, Nonl. Anal. TMA., 17 (1991), pp. 803-809.

14. J.S. Guo and B.Hu, The profile near the quenching time for the solution of a singular semilinear heat equation, Proc. Edin. Math. Soc., 40 (1997), pp. 437-456.

15. B. S. Jovanovic and L. G. Vulkov, Numerical solution of hyperbolic transmission problem, Comp. Meth. in Applied Math., 8 (2008), pp. 374-385.

16. H. A. Levine, Quenching, nonquenching and beyond quenching for solutions of some parabolic equations, Annali Mat. Pura Appl., 155 (1990), pp. 243-260.

17. K. W. Liang, P. Lin, and R. C. E. Tan, Numerical solution of quenching problems using mesh-dependent variable temporal steps, Appl. Numer. Math., 57 (2007), pp. 791-800.

18. K.W.Liang, P. Lin, M.T. Ong, and R.C.E.Tan, A splitting moving mesh method for reactiondiffusion equations of quenching type, J. Comput. Phys., 215(2) (2006), pp. 757-777.

19. T. Nakagawa, Blowing up on the finite difference solution to $u_{t}=u_{x x}+u^{2}$, Appl. Math. Optim., 2 (1976), pp. 337-350.

20. D. Nabongo and T.K. Boni, Quenching for semidiscretization of a heat equation with a singular boundary condition, Asympt. Anal., To appear.

21. D. Nabongo and T.K.Boni, Quenching time of solutions for some nonlinear parabolic equations, An. St. Univ. Ovidius constanta, 16 (2008), pp. 87-102.

22. D. Nabongo and T.K. Boni, Numerical quenching for semilinear parabolic equations, Math. Mod. and Anal., 13 (2008), no. 4, pp. 521-538.

23. F. K. N'gohisse and T.K. Boni, Numerical blow-up solutions for some semilinear heat equations, Elect. Trans. Numer. Anal., 30 (2008), pp. 247-257.

24. P. Quittner and P. Souplet, Superlinear parabolic problems. Blow-up, global existence and steady states, Birk?user Verlag, Basel, Basel, (2007).

25. D. Phillips, Existence of solutions of quenching problems, Appl Anal., 24 (1987), pp. $253-264$.

26. Q. Sheng and A.Q. M. Khaliq, Adaptive algorithms for convection-diffusion-reaction equations of quenching type, Dyn. Contin. Discrete Impuls. Syst. Ser. A, Math. Anal., 8 (2001), pp. 129-148.

27. Q. Sheng and A. Q. M. Khaliq,A compound adaptive approach to degenerate nonlinear quenching problems, Numer. Methods PDE, 15 (1999), pp. 29-47.

28. F. B. Weissler, Single point blow-up of semilinear initial value problem, J. Diff. Equat., 55 (1984), pp. 202-224.

29. L. Zhang, Existence, uniqueness and exponential stability of traveling wave solutions of some integral differential equations arising from neuronal networks, J. Diff. Equat., 197 (2004), pp. 162-196. 